\title{
A temperature-modulated dilatometer by using a piezobender-based device
}

\author{
Cite as: Rev. Sci. Instrum. 91, 123901 (2020); doi: 10.1063/5.0010826 \\ Submitted: 17 April 2020 • Accepted: 10 November 2020 • \\ Published Online: 4 December 2020
}

\author{
Yanhong Gu, ${ }^{1,2}$ Bo Liu, ${ }^{1,2}$ Wenshan Hong, ${ }^{1,2}$ Zhaoyu Liu, ${ }^{1,3}$ Wenliang Zhang, ${ }^{1,4}$ Xiaoyan Ma, ${ }^{1,2, a)}$ \\ and Shiliang $\mathrm{Li}^{1,2,5, \mathrm{~b})}(\mathbb{D}$
}

\author{
AFFILIATIONS \\ ${ }^{1}$ Beijing National Laboratory for Condensed Matter Physics, Institute of Physics, Chinese Academy of Sciences, \\ Beijing 100190, China \\ ${ }^{2}$ School of Physical Sciences, University of Chinese Academy of Sciences, Beijing 100190, China \\ ${ }^{3}$ Department of Physics, University of Washington, Seattle, Washington 98195, USA \\ ${ }^{4}$ Swiss Light Source, Paul Scherrer Institut, CH-5232 Villigen PSI, Switzerland \\ ${ }^{5}$ Songshan Lake Materials Laboratory, Dongguan, Guangdong 523808, China
}

a) Electronic mail: mxy@iphy.ac.cn

b) Author to whom correspondence should be addressed: slli@iphy.ac.ch

\begin{abstract}
We report a new design of a temperature-modulated dilatometer, which obtains the linear thermal expansion coefficient by measuring the oscillating changes of the sample's length and temperature by using a piezobender and a thermocouple, respectively. Using an iron-based superconductor $\mathrm{KFe}_{2} \mathrm{As}_{2}$ as an example, we show that this device is able to measure thin samples with high resolutions at low temperatures and high magnetic fields. Despite its incapability of giving absolute values, the new dilatometer provides a high-resolution method to study many important physical properties in condensed matter physics, such as thermal and quantum phase transitions and vortex dynamics in the superconducting state. The prototype design of this device can be further improved in many aspects to meet particular requirements.
\end{abstract}

Published under license by AIP Publishing. https://doi.org/10.1063/5.0010826

\section{I. INTRODUCTION}

The thermal expansion of a material describes the variation in its length or volume with temperature and is a fundamental thermodynamical parameter in studying the physical properties of solids. In particular, studying the change in thermal expansion across a phase transition has become one of the important techniques in condensed matter physics since it can reflect the intrinsic change of the electronic system with very high resolution. ${ }^{2-6}$ In the case of studying superconductors, the thermal-expansion measurement can detect phase transitions and vortex dynamics within the superconducting state, ${ }^{7-10}$ which shows its unique advantages compared to resistivity and magnetic-susceptibility measurements. The ratio between the thermal expansion and the specific heat gives the Grüneisen parameter, which is a crucial thermodynamical parameter for studying quantum critical transitions. ${ }^{11-16}$
For low-temperature measurements in the field of condensed matter physics, the mostly used and accurate dilatometers are based on directly measuring the length change of a sample with temperature by a plate capacitor or based upon an atomic microscope piezocantilever. ${ }^{17-24}$ The linear thermal-expansion coefficient $\alpha$ can be directly derived from $d L / L d T$, where $L$ and $T$ are the length and temperature of the sample, respectively. Using the alternating-current (AC) heating method, the thermal expansion can also be measured by temperature-modulated dilatometers (TMDs), but previous reports only show measurements above room temperature. ${ }^{25-27}$ The advantage of TMDs is that the setup is simple. The difficulty is how to measure the alternating length change of a sample at low temperatures.

In this work, we present a new design of a TMD based on a piezobender device that can be used at low temperatures and high magnetic fields. It is based on the uniaxial pressure device, as 
reported previously. ${ }^{28,29}$ With slightly changing the setup, we find that the device can be easily adapted to measure the linear thermal expansion. This method is similar to temperature-modulated calorimetry (TMC) and other temperature-modulated measurements. $^{30-32}$ The basic principle is to periodically heat a sample so that the oscillating changes of a sample's length and temperature, i.e., $\Delta L$ and $\Delta T$, can be simultaneously determined with the assistance of a lock-in system. High resolutions can thus be achieved because of the lock-in system's capability of detecting small signals. The linear thermal expansion $\alpha$ can be directly obtained as $\Delta L / L \Delta T$. We will use the iron-based superconductor $\mathrm{KFe}_{2} \mathrm{As}_{2}$ as an example to show the ability of our device in measuring the thermal expansion at low temperatures and high magnetic fields.

\section{EXPERIMENTAL SETUP AND MEASUREMENT PRINCIPLES}

Figures 1(a) and 1(b) show the sketch and the photo of the AC dilatometer, respectively, which is similar to the uniaxial pressure device reported previously. ${ }^{28}$ It is composed of a piezobender and a sapphire block secured by a CuBe frame. The two ends of a thin-slab sample are glued on top of them by GE varnish. The length of the sample is limited by the adjustable distance between the piezobender and the sapphire, which can be reduced to $0.5 \mathrm{~mm}$ for our device. The model of the piezobender is NAC2222 (Noliac), with the length, width and thickness being $21 \mathrm{~mm}, 7.8 \mathrm{~mm}$, and $1.3 \mathrm{~mm}$, respectively. There are three contacts for the piezobender, and the two side ones are soldered together. By using it as a uniaxial pressure device, a DC voltage is applied to the piezobender, which will tend to move it due to the reverse piezoelectric effect and thus provide a force on the sample. To measure the thermal expansion, a heater and a thermometer are attached to two sides of the sample by $\mathrm{N}$ grease, as shown in Fig. 1(a). The heater can be an external heater such as a resistor heater, or the sample can be heated by itself with current flow. A thermocouple made of Ni90/Cr10 and $\mathrm{Au} 99.93 / \mathrm{Fe} 0.07$ wires was used as the thermometer. The setup shown in Fig. 1(a) was put on to the regular sample puck of the physical properties measurement system (PPMS, Quantum Design), as shown in Fig. 1(b), which provides a low-temperature and magneticfield environment. A lock-in system was used to provide the $\mathrm{AC}$ power and measure the signals from the thermometer and piezobender. The measurement diagram is shown in Fig. 1(c). In this work, we have used a two-channel lock-in system (OE1022D from SYSU Scientific Instruments) and two preamplifiers (SR560 from Stanford Research Systems).

Assuming that the AC current flowing though the heater with a resistance $R$ has the form $I_{A C} \sin \omega t$ and neglecting the phase change, the AC power supplied by it will be $P_{A C} \sin ^{2} \omega t$, where $P_{A C}=I_{A C}^{2} R / 2$. This will result in an oscillating temperature of the sample, $\Delta T$, with $2 \omega$ periodicity. With proper conditions, it has already been shown that the magnitude of $\Delta T$, i.e., $\Delta T_{A C}$, has the following form: ${ }^{30}$

$$
\Delta T_{A C}=\frac{P_{A C}}{2 \omega C_{s}}\left[1+\frac{1}{4 \omega^{2} \tau_{1}^{2}}+4 \omega^{2} \tau_{2}^{2}+\frac{2 K_{b}}{3 K_{s}}\right]^{-\frac{1}{2}},
$$

where $C_{s}$ is the heat capacity of the sample, $K_{b}$ is the thermal conductance between the sample and the bath, $K_{s}$ is the thermal conductance of the sample, $\tau_{1}$ is the relaxation time from the sample (a) Thermometer

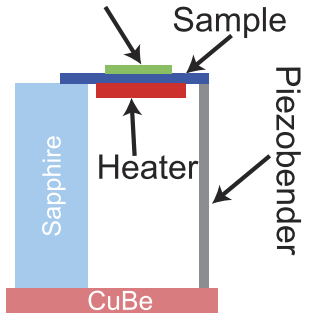

(c)
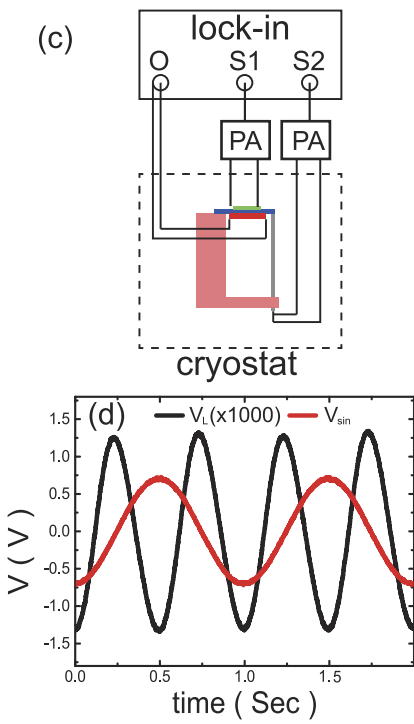

(b)

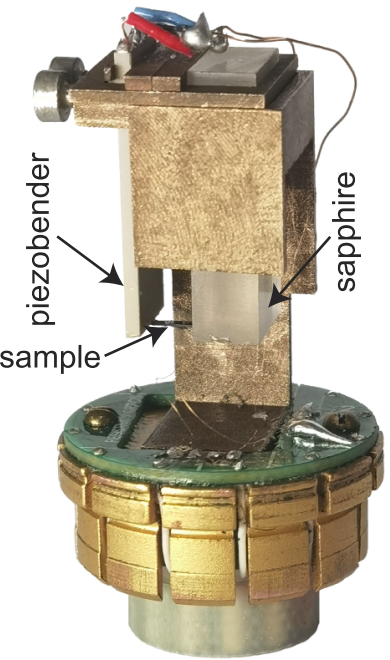

resistivity puck of PPMS

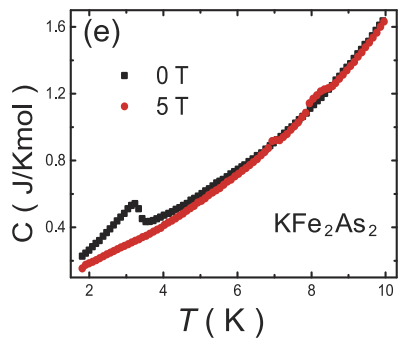

FIG. 1. (a) Sketch of the sample holder for the AC dilatometer. The two ends of the sample are attached to the sapphire block and piezobender, which are secured by a $\mathrm{CuBe}$ frame. A heater and a thermometer are attached to the two sides of the samples. (b) A photo of the AC dilatometer on the resistivity puck of the PPMS. The diameter of the puck is about $30 \mathrm{~mm}$. The bottoms of the piezobender and the sapphire are separated by several plates, whose thickness can be changed to fit the sample's length. (c) The measurement diagram of the AC dilatometer. $\mathrm{O}$, S1/S2, and PA represent the sine out and input channels of the lock-in amplifier and the pre-amplifier, respectively. (d) The comparison of the waveforms of $V_{L}$ (black line) and $V_{\text {sin }}$ (red line). The frequency of the sine out voltage of the lock-in amplifier is $1 \mathrm{~Hz}$. The temperature is $5 \mathrm{~K}$. A preamplifier with 1000 gain has been used. (e) Temperature dependence of the specific heat for $\mathrm{KFe}_{2} \mathrm{As}_{2}$ at 0 (black squares) and 5 (red circles) Tesla.

to the bath, and $\tau_{2}$ is associated with the time in which the sample, the heater, and the thermometer attain thermal equilibrium. The frequency-independent term $2 K_{b} / 3 K_{s}$ is the geometric correction due to the finite thermal diffusivity of the sample. In the case where $\tau_{1}$ is large while $\tau_{2}$ and $2 K_{b} / 3 K_{s}$ are small, $\Delta T_{A C}$ can take the much simpler form as

$$
\Delta T_{A C}=\frac{P_{A C}}{2 \omega C_{s}},
$$

which is the case for most traditional AC calorimeters. The oscillating temperature of the sample will simultaneously cause the oscillation of its volume. The change in the length for the setup shown in Fig. 1(a) along a particular direction can be measured by the piezobender since the movement of the top will create a voltage that 
can be detected by the lock-in system. Figure 1(d) shows an example where $V_{\sin }$ is the sine output voltage of the lock-in amplifier while $V_{P B}$ is the voltage of the piezobender. It is clear that the periodicity of $V_{P B}$ is twice that of $V_{\sin }$. Neglecting all the phase differences, the linear expansion coefficient $\alpha$ is thus

$$
\alpha=\frac{\Delta L_{A C}}{L_{0} \Delta T_{A C}}=\frac{\eta \kappa \Delta V_{L}}{L_{0} \Delta T_{A C}}
$$

where $L_{0}$ is the static length of the sample between the tops of the $\mathrm{BeCu}$ frame and the piezobender, which is assumed to be a constant value since its change with temperature can be neglected. The magnitude of the oscillating length, $\Delta L_{A C}$, is equal to $\eta \kappa \Delta V_{L}$, where $\Delta V_{L}$ is the magnitude of the oscillating voltage on the piezobender. The coefficient $\kappa$ is the relationship between the moving distance of the top of the piezobender and the resultant voltage, which has been independently determined to be about $(43.5+0.48 \mathrm{~T}) \mathrm{nm} / \mathrm{V}$ below $10 \mathrm{~K}$ with the DC voltage supply. ${ }^{33}$ The coefficient $\eta$ is introduced to account for other factors that may affect the determination of the length change. The value of $\eta$ is hard to determine, so we will give a rough estimation by comparing our results with those measured in the capacitive dilatometer.

The sample used here is an iron-based superconductor $\mathrm{KFe}_{2} \mathrm{As}_{2}$. At $T_{c}=3.4 \mathrm{~K}$, its thermal expansion along the a axis direction shows a drastic jump, changing from positive to negative value. ${ }^{6}$ We grew the $\mathrm{KFe}_{2} \mathrm{As}_{2}$ samples by the flux method, as reported previously. ${ }^{34}$ The specific heat shows a clear superconducting transition at $0 \mathrm{~T}$, which disappears at $5 \mathrm{~T}$, as shown in Fig. 1(e). Most of the thermal-expansion results were measured on a slice of the $\mathrm{KFe}_{2} \mathrm{As}_{2}$ sample, which was cut along the a axis with a length, width, and thickness of $4 \mathrm{~mm}, 0.85 \mathrm{~mm}$, and $0.14 \mathrm{~mm}$, respectively. Two electronic contacts were made on the sample using silver epoxy. Because the resistance of the sample is very small $\left(<10^{-3} \Omega\right)$, it is the contact resistances that actually work as the heater. Some other $\mathrm{KFe}_{2} \mathrm{As}_{2}$ samples have also been measured to study the effect of sample size for some parameters.

\section{RESULTS}

According to Eqs. (2) and (3), the magnitudes of voltages from both the thermocouple and the piezobender, $\Delta V_{T}$ and $\Delta V_{L}$, should be proportional to the square of the magnitude of the heating voltage $V_{\text {sin }}$. As shown in Figs. 2(a) and 2(b), the quadratic relation holds for small voltages. At large voltages, $\Delta V_{T}$ becomes lower than the value from linear extrapolation. This is most likely due to the DC heating effect, ${ }^{31}$ which results in an increase in the sample temperature and thus an increase in the specific heat and a decrease in $\Delta T$. To avoid this effect, $V_{\sin }$ is fixed at $0.3 \mathrm{~V}$ in the following measurements.

The frequency dependence of $\Delta V_{L}$ and $\Delta V_{T}$ can be well described by Eq. (1), neglecting the term $2 K_{b} / 3 K_{s}$, as shown in Figs. 2(c) and 2(d), respectively. At low temperatures ( $2 \mathrm{~K}$ and $4 \mathrm{~K}$ ), $f \Delta V_{T}$ becomes frequency-independent at high frequencies, which is because $\tau_{2}$ is very small $(\sim 2.1 \mathrm{~ms})$. With increasing temperatures, $\tau_{2}$ becomes larger so that its effect moves to lower frequencies, and there is no frequency-independent region. For $f \Delta V_{L}$, the frequencyindependent region exists at all temperatures. The difference in $\tau_{2}$ for $\Delta V_{L}$ and $\Delta V$ suggests that it takes a longer time for the thermocouple to attain thermal equilibrium since $\mathrm{N}$-grease has been used to attach it to the sample. The frequency-independent region means that the signal has the form in Eq. (2), which suggests that the device
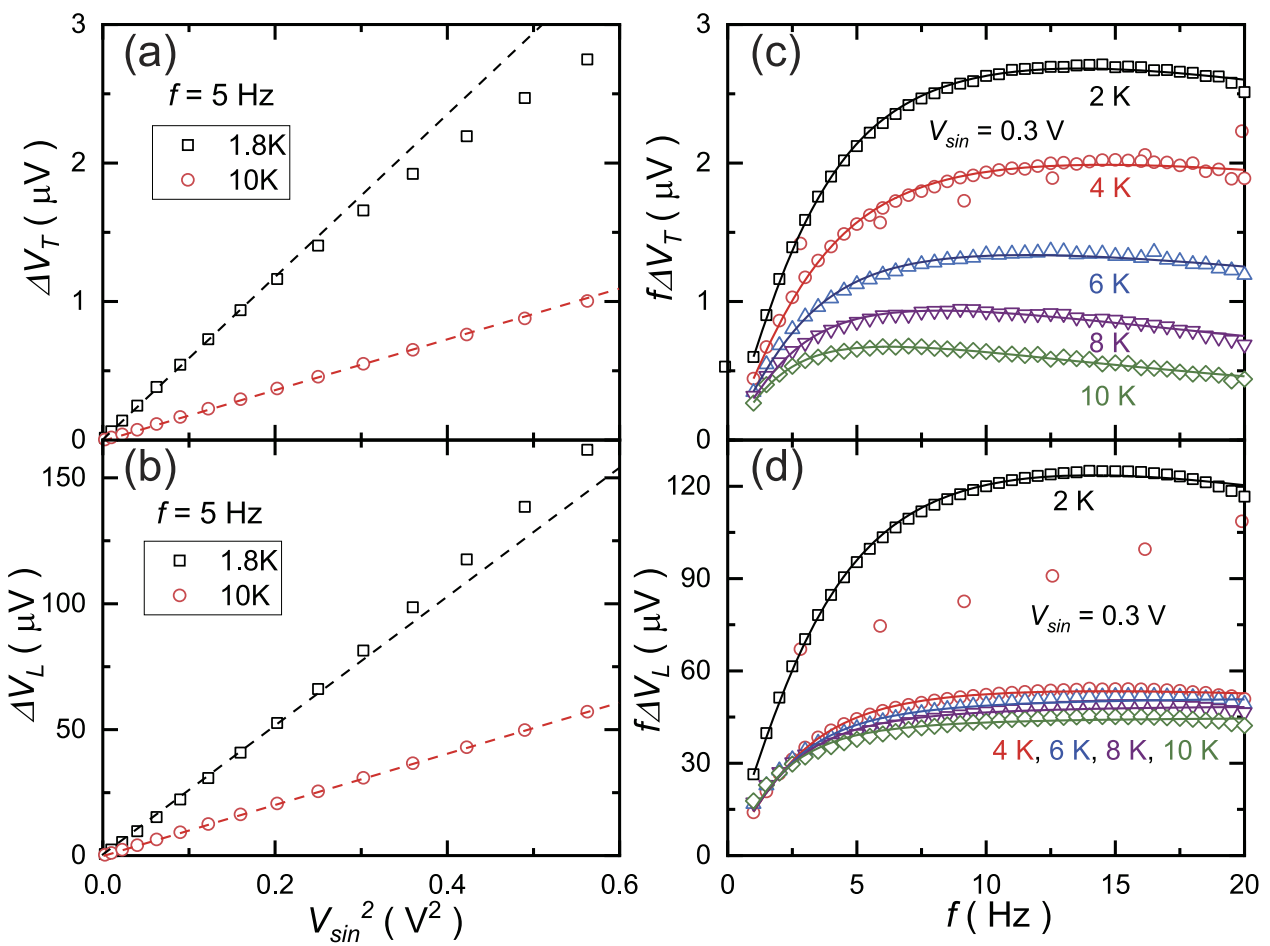

FIG. 2. (a) and (b) $V_{\text {sin }}^{2}$ dependence of $\Delta V_{T}$ and $\Delta V_{L}$, respectively. The frequency is fixed at $5 \mathrm{~Hz}$. The temperatures are $10 \mathrm{~K}$ and $1.8 \mathrm{~K}$. The dashed lines are fitted by a linear function for $V_{\text {sin }}<0.3 \mathrm{~V}$. (c) and (d) The frequency dependence of $f V_{T}$ and $f V_{L}$, respectively. The solid lines are the fitted results according to Eq. (1). 
works in the optimal condition for heat-capacity measurements in these frequencies.

Figure 3(a) shows the temperature dependence of $\Delta T$, which is calculated using $\Delta V_{T} / S$, where $S$ is the Seebeck coefficient of the thermocouple. It increases with decreasing temperature and shows a dip at $T_{c}$. According to Eq. (2), the specific heat should be proportional to $1 /(f \Delta T)$ if the power $P_{A C}$ has little temperature dependence. Figure $3(\mathrm{~b})$ shows the comparison between $1 /(f \Delta T)$ and the specific heat $C$. Below $5 \mathrm{~K}$, the data above $10 \mathrm{~Hz}$ closely follow the temperature dependence of $C$, which indicates the device is working at the optimal condition for specific heat measurements. On the other hand, significant deviation occurs at higher temperatures, which is due to the increase in $\tau_{2}$ for $\Delta V_{T}$, as discussed above.

Figure 3(c) shows the temperature dependence of $\Delta V_{L}$, which shows a very sharp dip at $T_{c}$ for all frequencies. This is because the linear thermal expansion coefficient $\alpha$ along the a axis changes sign at $T_{c}{ }^{6}$ As shown in Fig. $3(\mathrm{~d})$, the phase $\theta_{L}$ for $\Delta V_{L}$ changes exactly by $180^{\circ}$ at $T_{c}$, which demonstrates the sign change of $\Delta L$ across $T_{c}$. Except this 180-degree change, the value of $\theta_{L}$ has no physical meaning, so the change in length is only associated with $\Delta V_{L}$.

Figure 3(e) shows the linear thermal expansion $\alpha_{a}$ along the a axis obtained by Eq. (3) with $\eta=134$. The value of $\eta$ is obtained by comparing our results with previous results measured by the capacitive dilatometer. ${ }^{6}$ The origin of the large value of $\eta$ will be discussed later. The temperature dependence of $\alpha_{a}$ below $5 \mathrm{~K}$ shows almost no frequency dependence and can be nicely normalized to the reported values. With increasing temperature, $\alpha_{a}$ becomes different for different frequencies, which is because the frequency dependence of $\Delta V_{L}$ and $\Delta V_{T}$ becomes different, as shown in Figs. 2(c) and 2(d).

Figure 3(f) shows the temperature dependence $f \Delta L=f \eta \kappa \Delta V_{L}$ at different frequencies. The reason to plot this is that this value should be proportional to the linear Grüneisen parameter $\Gamma_{a}=\alpha_{a} / C_{s}$, according to Eqs. (1) and (3). It is clear that the calculated values nicely follow the temperature dependence of $\Gamma_{a}$ calculated from $\alpha_{a}$
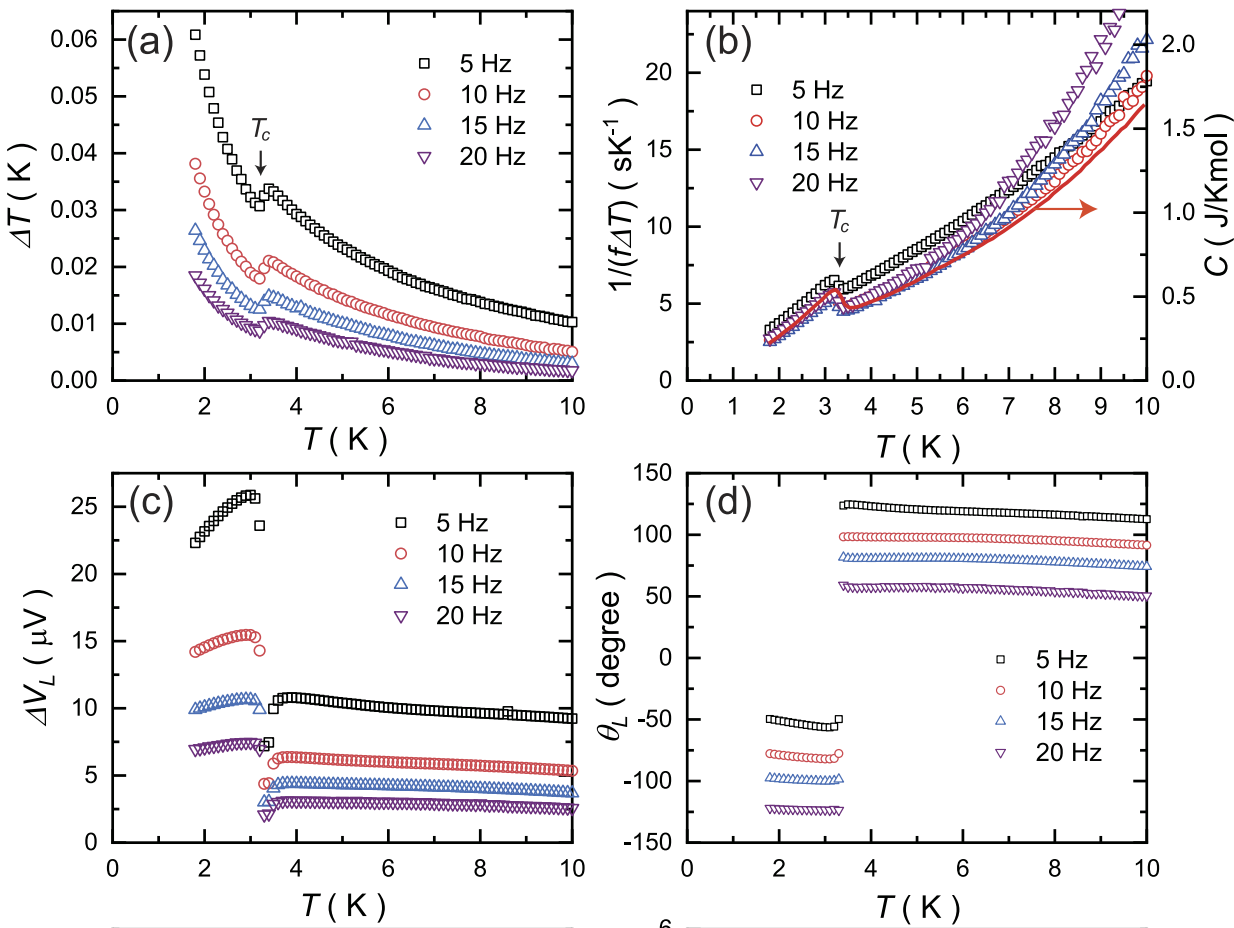

FIG. 3. (a) Temperature dependence of $\Delta T$. (b) Temperature dependence of $1 /(f \Delta T)$ and the specific heat (solid line). (c) and (d) Temperature dependence of $\Delta V_{L}$ and $\theta_{L}$, respectively. (e) The temperature dependence of the linear temperature thermal expansion along the a axis $\alpha_{a}$. The solid line is $\alpha_{a}$ from Ref. 6 . (f) The temperature dependence of the linear Grüneisen parameter. The solid line in (d) is $\Gamma_{a}$ calculated by $\alpha$ from Ref. 6 and the specific heat of $\mathrm{KFe}_{2} \mathrm{As}_{2}$.
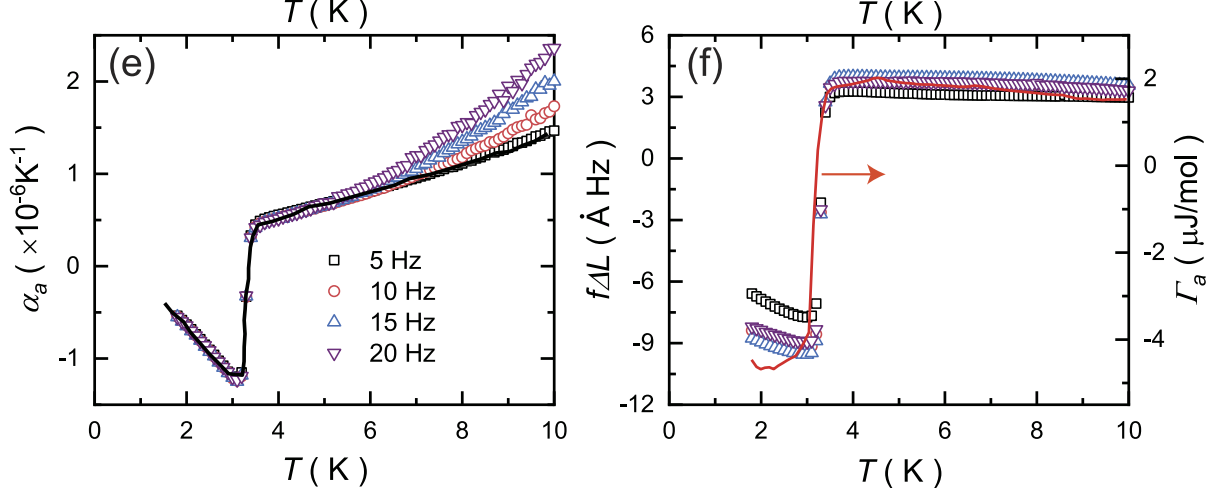
in Ref. 6 and the specific heat. It should be noted that in this method, there is actually no need to measure $\Delta T$. The absolute value of $\Gamma_{a}$ can be obtained if the power $P_{A C}$ can be determined.

\section{DISCUSSIONS}

The above-mentioned results demonstrate that it is possible to measure the thermal expansion at low temperatures by the $\mathrm{AC}$ method based on a piezobender device. There are several advantages compared to the capacitive dilatometer. First, the design and fabrication of the sample holder and the experimental setup are very simple. The capacitive low-temperature dilatometer, on the other hand, is very specialized, and its measurement needs a high-resolution capacitance meter. Second, the device here can measure very thin crystals, which may be crucial for some materials where only thin slices of crystals are available. With the known spring constant of the piezobender $(\sim 0.0308 \mathrm{~N} / \mu \mathrm{m})$ and Young's modulus $(\sim 105 \mathrm{GPa}$ along the a axis for $\mathrm{KFe}_{2} \mathrm{As}_{2}{ }^{35}$ ), we estimate that $F / F^{\prime}$ is about 100 for the sample used here, where $F$ and $F^{\prime}$ are the force created by the thermal expansion of the sample and the force required to move the tip of the piezobender, respectively. This means that although the sample is thin, its thermal expansion is able to push the piezobender and create the voltage to be measured. In fact, the thinnest sample we have tried is about $18 \mu \mathrm{m}$, which still has $F / F^{\prime} \approx 30$. Third, the resolution is good, as shown in Figs. 3(e) and 3(f). Based on the relative noise level of $\Delta V_{L}\left(\sim 10^{-4}\right)$ and the value of $\alpha_{a}$, we estimate that the absolute resolution to resolve the length change in the sample is about $10^{-4} \AA$ at $2 \mathrm{~K}$. The resolution for $\alpha$ in this work is about 0.5 $\times 10^{-9} \mathrm{~K}^{-1}$ at $2 \mathrm{~K}$. It should be pointed out that a better resolution can be achieved in the future by introducing better shielding and design of the electrical circuits and different choices of the heater, the piezobender, and the thermometer. Ideally, one may finally achieve a resolution better than $10^{-4} / \sqrt{f} \AA$ at $2 \mathrm{~K}$ for the length change if the noise level of the lock-in system is about $2 \mathrm{nV} / \sqrt{f}$.

As shown by this work, it is also possible to obtain the specific heat by our device, as shown in Fig. 3(b). However, whether this is possible seems to depend on the sample thickness (and thus the mass); as for much thinner samples $(\sim 10 \mu \mathrm{m})$, the measured $C$ is about 10 times larger than the actual value, which may be because a large amount of heating power is applied on other parts of the device. Nevertheless, the oscillating temperature of the sample can still be represented by $\Delta T$, so the $\alpha$ value is still reliable. The simultaneous measurements of the linear thermal expansion $\alpha$ and the specific heat $C_{s}$ of a sample mean that one can obtain the linear Grüneisen parameter $\Gamma \propto \alpha / C_{s}$. In principle, even without the knowledge of $\Delta T$ and hence the specific heat, it is still possible to directly obtain $\Gamma$ $\propto \Delta V_{L}$, according to Eqs. (2) and (3), as shown in Fig. 3(f), although it is still required that the measured $C$ is close to $C_{s}$. We'd like to point it out although that in some cases where both $C_{s}$ and $\Gamma$ diverge, such as at a quantum critical point, $\Delta V_{L}$ still provides a good approximation to $\Gamma$.

There are several disadvantages for the TMD device here. First, it is hard to obtain the absolute value of $\alpha$. As shown above, the values measured here are more than two orders smaller than the actual ones. We have found that the relaxation time of the charges in the piezobender, which is about $20 \mathrm{~ms}$ at $2 \mathrm{~K}$, makes the signal 4 times smaller than the maximum voltage created. Moreover, the output voltage of a piezobender strongly depends on the frequency $f$ and becomes much smaller when $f$ is away from the resonance frequency, ${ }^{36-38}$ which is about $1300 \mathrm{~Hz}$ for the piezobender used here. Fortunately, our results in Fig. 3(f) show that for a fixed setup, $\eta$ is independent of sample size, which means that the absolute value of $\alpha$ can be obtained by carefully comparing the results between the TMD and capacitive dilatometers. In fact, for the several $\mathrm{KFe}_{2} \mathrm{As}_{2}$ samples we have measured, the values of $\eta$ are all around 140. Second, the device works well below $5 \mathrm{~K}$ where the optimal working condition described by Eq. (2) is satisfied. At higher temperatures, the effect of $\tau_{2}$ on $\Delta T$ becomes significant, which results in the deviation of $\alpha_{a}$ from the linear temperature dependence [Fig. 3(e)]. It is interesting to note that for $\Delta V_{L}$, there is always a region that satisfies the optimal working condition, as shown in Fig. 2(d). Consequently, $\tau_{2}$ of the sample, i.e., the time in which the sample attains thermal equilibrium, is much smaller than that of the thermocouple. If a thermometer with a smaller heat capacity and better thermal conductance can be used, this issue may be solved.

Despite the above-mentioned disadvantages, the TMD introduced here can be used to study some important physical properties, such as the phase transitions and vortex dynamics in the superconducting state. In particular, since the device works well below $5 \mathrm{~K}$, it may be possible to apply it below $1.8 \mathrm{~K}$ to study quantum phase transitions. Moreover, although it is hard to obtain the absolute value, studying the systematic change of the thermal expansion in a particular system with doping and magnetic field is still reliable.

\section{SUMMARY}

We have designed a temperature-modulated dilatometer based on a piezobender device, which can measure the linear thermal expansion coefficient and, in principle, the Grüneisen parameter. Although it is hard to obtain the absolute values, the device has the capability of measuring very thin samples with high resolutions, as illustrated by measuring $\mathrm{KFe}_{2} \mathrm{As}_{2}$ single crystals. Considering that this device is still a prototype, a lot of improvements should be possible in the future.

Note added in proof: After the acceptance of the paper, we have found out that the signal will increase 35 times if the high dynamic reserve is set for our lock-in system, which perfectly explains the value of $\eta$.

\section{ACKNOWLEDGMENTS}

We thank Professor Jing Guo for his help. This work was supported by the National Key R\&D Program of China (Grant Nos. 2017YFA0302903 and 2016YFA0300502), the National Natural Science Foundation of China (Grant Nos. 11874401 and 11674406), and the Strategic Priority Research Program (B) of the Chinese Academy of Sciences (Grant Nos. XDB25000000, XDB07020000, and XDB28000000).

\section{DATA AVAILABILITY}

The data that support the findings of this study are available from the corresponding authors upon reasonable request.

\section{REFERENCES}

${ }^{1}$ T. H. K. Barron, J. G. Collins, and G. K. White, “Thermal expansion of solids at low temperatures," Adv. Phys. 29, 609 (1980). 
${ }^{2}$ G.-m. Zhao, M. B. Hunt, and H. Keller, "Strong oxygen-mass dependence of the thermal-expansion coefficient in the manganites $\left(\mathrm{La}_{1-x} \mathrm{Ca}_{x}\right)_{1-y} \mathrm{Mn}_{1-y} \mathrm{O}_{3}$," Phys. Rev. Lett. 78, 955-958 (1997).

${ }^{3}$ A. Bianchi, R. Movshovich, N. Oeschler, P. Gegenwart, F. Steglich, J. D. Thompson, P. G. Pagliuso, and J. L. Sarrao, "First-order superconducting phase transition in CeCoIn 5 ," Phys. Rev. Lett. 89, 137002 (2002).

${ }^{4}$ G. Motoyama, T. Nishioka, and N. K. Sato, "Phase transition between hidden and antiferromagnetic order in $\mathrm{URu}_{2} \mathrm{Si}_{2}$," Phys. Rev. Lett. 90, 166402 (2003).

${ }^{5}$ J. Hemberger, H.-A. K. von Nidda, V. Tsurkan, and A. Loidl, "Large magnetostriction and negative thermal expansion in the frustrated antiferromagnet $\mathrm{ZnCr}_{2} \mathrm{Se}_{4}$," Phys. Rev. Lett. 98, 147203 (2007).

${ }^{6}$ F. Hardy, A. E. Böhmer, D. Aoki, P. Burger, T. Wolf, P. Schweiss, R. Heid, P. Adelmann, Y. X. Yao, G. Kotliar, J. Schmalian, and C. Meingast, "Evidence of strong correlations and coherence-incoherence crossover in the iron pnictide superconductor $\mathrm{KFe}_{2} \mathrm{As}_{2}$," Phys. Rev. Lett. 111, 027002 (2013).

${ }^{7}$ R. Modler, P. Gegenwart, M. Lang, M. Deppe, M. Weiden, T. Lühmann, C. Geibel, F. Steglich, C. Paulsen, J. L. Tholence, N. Sato, T. Komatsubara, Y. Ōnuki, M. Tachiki, and S. Takahashi, "First-order transition between weak and strong pinning in clean superconductors with enhanced spin susceptibility," Phys. Rev. Lett. 76, 1292-1295 (1996).

${ }^{8}$ R. Lortz, C. Meingast, U. Welp, W. K. Kwok, and G. W. Crabtree, "Crystal-lattice coupling to the vortex-melting transition in $\mathrm{YBa}_{2} \mathrm{Cu}_{3} \mathrm{O}_{7-\delta}$," Phys. Rev. Lett. 90, 237002 (2003)

${ }^{9}$ S. Zaum, K. Grube, R. Schäfer, E. D. Bauer, J. D. Thompson, and H. v. Löhneysen, "Towards the identification of a quantum critical line in the $(p, B)$ phase diagram of CeCoIn 5 with thermal-expansion measurements," Phys. Rev. Lett. 106, 087003 (2011).

${ }^{10}$ H. K. Mak, P. Burger, L. Cevey, T. Wolf, C. Meingast, and R. Lortz, "Thermodynamic observation of a vortex melting transition in the Fe-based superconductor $\mathrm{Ba}_{0.5} \mathrm{~K}_{0.5} \mathrm{Fe}_{2} \mathrm{As}_{2}$," Phys. Rev. B 87, 214523 (2013).

${ }^{11}$ L. Zhu, M. Garst, A. Rosch, and Q. Si, "Universally diverging Grüneisen parameter and the magnetocaloric effect close to quantum critical points," Phys. Rev. Lett. 91, 066404 (2003).

${ }^{12}$ T. Lorenz, O. Heyer, M. Garst, F. Anfuso, A. Rosch, C. Rüegg, and K. Krämer, "Diverging thermal expansion of the spin-ladder system $\left(\mathrm{C}_{5} \mathrm{H}_{12} \mathrm{~N}\right)_{2} \mathrm{CuBr}_{4}$," Phys. Rev. Lett. 100, 067208 (2008).

${ }^{13}$ J. G. Donath, F. Steglich, E. D. Bauer, J. L. Sarrao, and P. Gegenwart, "Dimensional crossover of quantum critical behavior in CeCoIn 5 ," Phys. Rev. Lett. 100, 136401 (2008).

${ }^{14}$ C. Meingast, F. Hardy, R. Heid, P. Adelmann, A. Böhmer, P. Burger, D. Ernst, R. Fromknecht, P. Schweiss, and T. Wolf, "Thermal expansion and grüneisen parameters of $\mathrm{Ba}\left(\mathrm{Fe}_{1-x} \mathrm{Co}_{x}\right)_{2} \mathrm{As}_{2}$ : A thermodynamic quest for quantum criticality," Phys. Rev. Lett. 108, 177004 (2012).

${ }^{15}$ Y. Tokiwa, E. D. Bauer, and P. Gegenwart, "Zero-field quantum critical point in CeCoIn 5 ," Phys. Rev. Lett. 111, 107003 (2013).

${ }^{16}$ A. Steppke, R. Küchler, S. Lausberg, E. Lengyel, L. Steinke, R. Borth, T. Lühmann, C. Krellner, M. Nicklas, C. Geibel, F. Steglich, and M. Brando, "Ferromagnetic quantum critical point in the heavy-fermion metal $\mathrm{YbNi}_{4}\left(\mathrm{P}_{1-x} \mathrm{As}_{x}\right)_{2}$," Science 339, 933-936 (2013).

${ }^{17}$ G. M. Schmiedeshoff, A. W. Lounsbury, D. J. Luna, S. J. Tracy, A. J. Schramm, S. W. Tozer, V. F. Correa, S. T. Hannahs, T. P. Murphy, E. C. Palm, A. H. Lacerda, S. L. Bud'ko, P. C. Canfield, J. L. Smith, J. C. Lashley, and J. C. Cooley, "Versatile and compact capacitive dilatometer," Rev. Sci. Instrum. 77, 123907 (2006).

${ }^{18}$ R. Küchler, T. Bauer, M. Brando, and F. Steglich, "A compact and miniaturized high resolution capacitance dilatometer for measuring thermal expansion and magnetostriction," Rev. Sci. Instrum. 83, 095102 (2012).

${ }^{19} \mathrm{~S}$. Abe, F. Sasaki, T. Oonishi, D. Inoue, J. Yoshida, D. Takahashi, H. Tsujii, H. Suzuki, and K. Matsumoto, "A compact capacitive dilatometer for thermal expansion and magnetostriction measurements at millikelvin temperatures," Cryogenics 52, 452 (2012).
${ }^{20}$ R. S. Manna, B. Wolf, M. de Souza, and M. Lang, "High-resolution thermal expansion measurements under helium-gas pressure," Rev. Sci. Instrum. 83, 085111 (2012).

${ }^{21}$ D. Inoue, D. Kaido, Y. Yoshikawa, M. Minegishi, K. Matsumoto, and S. Abe, "Thermal expansion and magnetostriction measurements using a high sensitive capacitive dilatometer at millikelvin temperatures," J. Phys.: Conf. Ser. 568, 032001 (2014).

${ }^{22}$ R. Küchler, C. Stingl, and P. Gegenwart, "A uniaxial stress capacitive dilatometer for high-resolution thermal expansion and magnetostriction under multiextreme conditions," Rev. Sci. Instrum. 87, 073903 (2016).

${ }^{23}$ R. Küchler, A. Wörl, P. Gegenwart, M. Berben, B. Bryant, and S. Wiedmann, "The world's smallest capacitive dilatometer for high-resolution thermal expansion and magnetostriction in high magnetic fields," Rev. Sci. Instrum. 88, 083903 (2017).

${ }^{24}$ L. Wang, G. M. Schmiedeshoff, D. E. Graf, J.-H. Park, T. P. Murphy, S. W. Tozer, E. Palm, J. L. Sarrao, and J. C. Cooley, "Application of an atomic force microscope piezocantilever for dilatometry under extreme conditions," Meas. Sci. Technol. 28, 065006 (2017).

${ }^{25} \mathrm{~K}$. Uchino and L. E. Cross, "A very high sensitivity AC dilatometer for the direct measurement of piezoelectric and electrostrictive constants," Ferroelectrics $\mathbf{2 7}, 35$ (1980).

${ }^{26} \mathrm{~T}$. H. Johansen, “An AC dilatometer for linear expansivity measurement," High Temp. High Pressure 19, 77 (1987).

${ }^{27}$ J. Su, P. Moses, and Q. M. Zhang, "A bimorph based dilatometer for field induced strain measurement in soft and thin free standing polymer films," Rev. Sci. Instrum. 69, 2480 (1998).

${ }^{28}$ Z. Liu, Y. Gu, W. Zhang, D. Gong, W. Zhang, T. Xie, X. Lu, X. Ma, X. Zhang, R. Zhang, J. Zhu, C. Ren, L. Shan, X. Qiu, P. Dai, Y.-f. Yang, H. Luo, and S. Li, "Nematic quantum critical fluctuations in $\mathrm{BaFe}_{2-x} \mathrm{Ni}_{x} \mathrm{As}_{2}$," Phys. Rev. Lett. 117, 157002 (2016).

${ }^{29}$ Y. Gu, Z. Liu, T. Xie, W. Zhang, D. Gong, D. Hu, X. Ma, C. Li, L. Zhao, L. Lin, Z. Xu, G. Tan, G. Chen, Z. Y. Meng, Y.-f. Yang, H. Luo, and S. Li, "Unified phase diagram for iron-based superconductors," Phys. Rev. Lett. 119, 157001 (2017).

${ }^{30}$ P. F. Sullivan and G. Seidel, "Steady-state, ac-temperature calorimetry," Phys Rev. 173, 679-685 (1968).

${ }^{31}$ E. Gmelin, "Classical temperature-modulated calorimetry: A review," Thermochim. Acta 304-305, 1 (1997).

${ }^{32}$ M. S. Ikeda, J. A. W. Straquadine, A. T. Hristov, T. Worasaran, J. C. Palmstrom, M. Sorensen, P. Walmsley, and I. R. Fisher, "AC elastocaloric effect as a probe for thermodynamic signatures of continuous phase transitions," Rev. Sci. Instrum. 90, 083902 (2019).

${ }^{33} \mathrm{X}$. Ma and S. Li (unpublished).

${ }^{34}$ Z. Liu, Y. Gu, W. Hong, T. Xie, D. Gong, X. Ma, J. Liu, C. Hu, L. Zhao, X. Zhou, R. M. Fernandes, Y.-f. Yang, H. Luo, and S. Li, "Nonlinear uniaxial pressure dependence of $T_{c}$ in iron-based superconductors," Phys. Rev. Res. 1, 033154 (2019).

${ }^{35}$ F. F. Tafti, J. P. Clancy, M. Lapointe-Major, C. Collignon, S. Faucher, J. A. Sears, A. Juneau-Fecteau, N. Doiron-Leyraud, A. F. Wang, X.-G. Luo, X. H. Chen, S. Desgreniers, Y.-J. Kim, and L. Taillefer, "Sudden reversal in the pressure dependence of $T_{c}$ in the iron-based superconductor $\mathrm{CsFe}_{2} \mathrm{As}_{2}$ : A possible link between inelastic scattering and pairing symmetry," Phys. Rev. B 89, 134502 (2014).

${ }^{36}$ S. Roundy and P. K. Wright, "A piezoelectric vibration based generator for wireless electronics," Smart Mater. Struct. 13, 1131 (2004).

${ }^{37}$ J. Ajitsaria, S. Y. Choe, D. Shen, and D. J. Kim, "Modeling and analysis of a bimorph piezoelectric cantilever beam for voltage generation," Smart Mater. Struct. 16, 447 (2007).

${ }^{38}$ E. K. Reilly, F. Burghardt, R. Fain, and P. Wright, "Powering a wireless sensor node with a vibration-driven piezoelectric energy harvester," Smart Mater. Struct. 20, 125006 (2011). 